\title{
Structure determination of the mature Usutu SAAR-1776 virus using single particle cryo-electron microscopy
}

Baldeep Khare ${ }^{1}$, Thomas Klose ${ }^{1}$, Qianglin Fang ${ }^{2}$, Michael Rossmann ${ }^{1}$ and Richard Kuhn ${ }^{3}$

${ }^{1}$ Purdue University, United States, ${ }^{2}$ University of California Berkeley, United States, ${ }^{3}$ Purdue University, West Lafayette, Indiana, United States

Usutu virus (USUV), an arbovirus belonging to the JEV serocomplex of family Flaviviridae, is currently rampant in Europe and increasingly recognized for its pervasive asymptomatic presence in the human population. Originally identified in Africa, USUV was introduced into Europe by migratory birds. In rare cases, USUV infection in humans leads to severe disease that involves encephalitis and meningoencephalitis. However, no preventative measures or treatment options exist for treating human USUV infections. Furthermore, relatively little is known about the biology of the USUV virus and the underlying mechanisms of pathogenesis in humans. To fill this gap in our knowledge-base, we determined two structures of the mature USUV virus using single particle cryo-electron microscopy to a resolution of $2.4 \AA$. The mature virus particle displays an icosahedral arrangement of 180 copies each of the two structural proteins, the envelope glycoprotein $(\mathrm{E})$ and the membrane protein. The findings span new knowledge in two areas: high-resolution features not previously observed in flavivirus E proteins and structural features unique to USUV. Key findings from the two structures reveal: 1) presence of a fusion loop disulfide bond in one of the monomers of the asymmetric subunit in one structure, revealing asymmetry in the fusion loop of USUV; 2) presence of at least two virus populations in the sample, distinguished after structure determination but not during particle classification; 3) lipid densities observed at three sites, including two involving lipid head group interactions with the E stem, revealing key residues involved in interaction with the bilayer; and 4) a secondary structure harboring the second, unique glycosylation site in the USUV E protein, and potential residues that may confer GAG binding and hence explain the broad cellular tropism observed for the virus. Our findings provide structural information to understand USUV pathogenesis as well as expands the purview of flavivirus biology.

References

- Gaibani, P. and G. Rossini, An overview of Usutu virus. Microbes Infect, 2017. 19(7-8): p. 382-387.

- Cle, M., et al., Usutu virus: A new threat? Epidemiol Infect, 2019. 147: p. e232.

- Weissenbock, H., et al., Emergence of Usutu virus, an African mosquito-borne flavivirus of the Japanese encephalitis virus group, central Europe. Emerg Infect Dis, 2002. 8(7): p. 652-6. 